\title{
A web-based service for improving conformance to medication treatment and patient-physician relationship
}

\author{
Nikolaos Riggos, Ilias Skalkidis, George Karkalis, Maria Haritou, Dimitris \\ Koutsouris \\ Biomedical Engineering Laboratory \\ National Technical University of Athens, \\ 9 Iroon Polytechniou Str., Zografou Campus, 15773 \\ Athens, Greece \\ Tel: $(+30) 210-7722269$ \\ nriggos@biomed.ntua.gr \\ WWW home page: http://www.biomed.ntua.gr
}

\begin{abstract}
The proposed service is a health-related web portal serving a twofold purpose: Reminding the patient of important tasks related to a longterm treatment, such as medication intake, clinical encounters, follow-up actions etc., as well as tightening the patient-physician relationship through bidirectional communication. The physician is able to register and review patient-related information through a user-friendly web-based interface, assign medication treatment plans, examination plans etc. The patient receives notifications about the treatment plan through several means, including Short Message Service (SMS) and e-mail. The common web-based space shared by the patient and the physician enables the bidirectional exchange of observations or comments about the treatment, publishing additional guidelines augmenting the treatment outcome, reporting side-effects etc. The implementation of the service is based on MS SQL Server Notification Services technology and we believe that is has the potential to improve adherence to the medication plans, especially in the case of long-term therapy.
\end{abstract}

\section{Introduction}

The success of drugs in controlling many of the illnesses associated with old age, means that people can continue to enjoy full and independent lives in the community providing that they comply with their prescribed medications. Unfortunately, many older people suffer from a number of different chronic conditions each of which requires a separate type of medication. Consequently, significant numbers need to

Please use the following format when citing this chapter:

Riggos, N., Skalkidis, 1., Karkalis, G., Haritou, M., Koutsouris, D., 2007, in IFIP International Federation for Information Processing, Volume 247, Artificial Intelligence and Innovations 2007: From Theory to Applications, eds. Boukis, C., Pnevmatikakis, L., Polymenakos, L., (Boston: Springer), pp. 157-162. 
administer several drugs during the course of a day. In an institutional care setting such as a hospital or nursing home, responsibility for taking the correct medication at the correct time lies with the medical and nursing personnel. When the patient returns home, professional help is not generally available at the times when the drugs must be administered. There is therefore a risk that the medication may be forgotten, the wrong dose will be taken or that the wrong medication will be taken. These risks are especially relevant to older people whose cognitive abilities may be diminishing and those who are suffering from any degree of confusion.

Despite wide recognition and documentation of its existence, patient noncompliance to prescribed medication regimens continues to be a prevalent problem. Stewart and Cluff have stated that the percentage of patients making medication errors in the selfadministration of prescribed medications, with few exceptions, has ranged between 25 and 95 per cent [1]. The most frequently cited errors relate to dosage, frequency of administration, and drug identification. Several studies have shown that over half of elderly patients do not take their drugs as prescribed, $[2,3]$ and the percentage of all patients who make errors is probably between $25 \%$ and $59 \%$ [1]. Many of these patients may not clearly understand their regimens, [4] and about $4-35 \%$ of patients misuse their drugs to such an extent that they endanger their health [1]. Furthermore, the lack of effective communication between physicians and patients about medications may be an important reason why patients do not follow medical advice [5]. According to Kanjanarat et. al., "Most preventable adverse drug events occur in the prescribing stage of the medication-use process and have been attributed to inappropriate prescribing decisions and inappropriate monitoring" [6]. It is therefore evident that a complete solution that helps both the physicians and the patients reduce medication errors is needed.

The application presented at this paper is a user friendly web based portal, designed specifically to provide reminder services to the patient as well as monitoring and archiving services to the physician tightening the patient-physician relationship through bidirectional communication.

\section{Application Description}

The most appropriate way to describe this application is by describing the roles of the three distinct users of the system.

\subsection{The administrator}

The administrator is responsible for the administration of all the users and the system in general. The administrator is the only user that can create a "doctor user", change a user password or delete a user. 


\subsection{The Doctor}

The doctor has the option to create a user account for the patient and prescribe a specific medication from a list of drugs available to him (Figure 1). The doctor can edit a medication schedule, indicating the frequency of the drug intake, the dose and the specific time a reminding notification will be sent to his patient. He also has the option to choose whether the notification will be sent via an SMS and/or an e-mail. Additionally the doctor can create a new scheduled examination specifying the date and time this examination will occur, as well as the number of days he would like his patient to be notified in advance. Finally the doctor is able to add explanatory comments on every parameter of a medication schedule, making it easier for the patient to remember and follow his instructions.

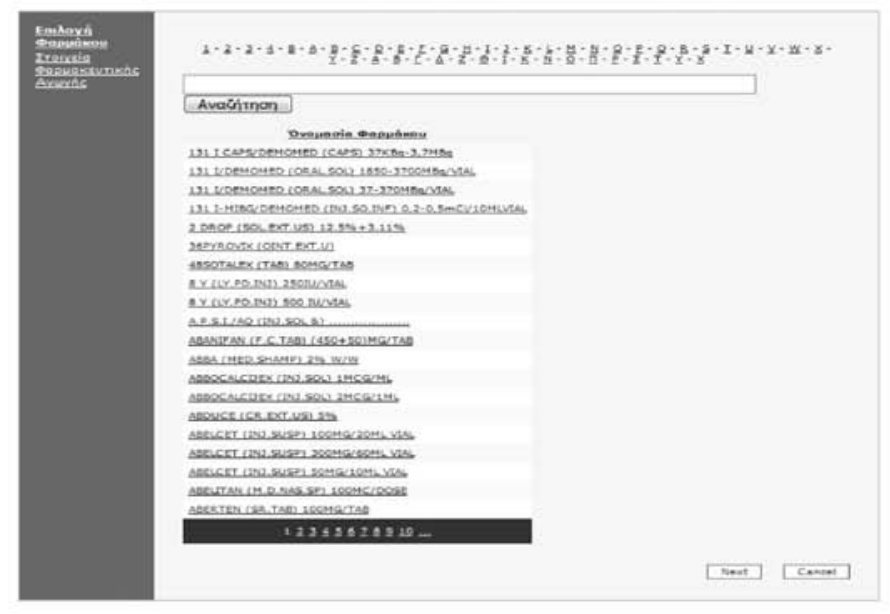

Figure 1. The Doctor can choose a drug from the database using a search engine

\subsection{The Patient}

When the patient logins into the system, he can view a summary of the information related to him. These include the doses of drugs to be taken in the next 24 hours, all active medication regimens, a full history of medication plans completed and any scheduled appointments created by the doctor. Everything is presented in a clear and concise manner with extra detail coming up to the screen if a specific element is clicked (Figure 2). The patient can insert comments regarding a specific medication plan, drug or dose at any time, providing feedback to his doctor. If any new entry is recorded a notification is sent to the doctor. 


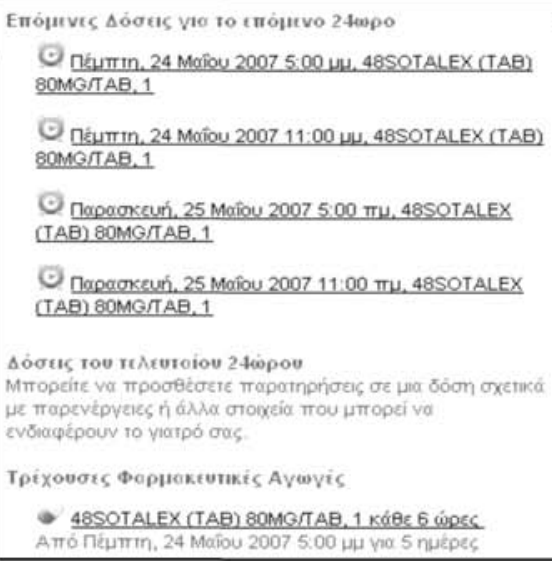

Figure 2. The patient details screen

\section{System Architecture}

The system architecture is largely based on MS SQL Server Notification Services technology [8] (Figure 3). Notification Services is a programming framework for building applications that send notifications to subscribed users, and a server that hosts the applications. The user is required to subscribe to the service by specifying triggering events when the notification should be generated and sent to the user. This is accomplished through a subscription management interface library, developed using subscription management objects supplied with Notification Services. A web service is built upon the subscription management interface exposing its functionality. The web application uses this web service wrapper to add user subscription information to the Notification Services Database. Notification Services stores the subscriber and delivery information in a central Notification Services' database and it stores individual subscriptions in application-specific databases. This allows applications to share the global subscriber data while separately storing subscriptions for each application.

Notification Services handle the final delivery of notifications through the use of delivery channels, which can be thought of as pipes to delivery services. The delivery channels package the notifications into protocol packets and then send them to the delivery service that handles the final delivery. An e-mail delivery channel is included as an internal component of the Notification Services and its functionality was used as provided by the framework. Additionally, Notification Services provide a mechanism for implementing custom delivery channels. Using this mechanism, a 
custom SMS delivery channel and an SMS Web Service were built in order to communicate with the Clickatell Bulk SMS Gateway, which handles the sending of the SMS to the users.

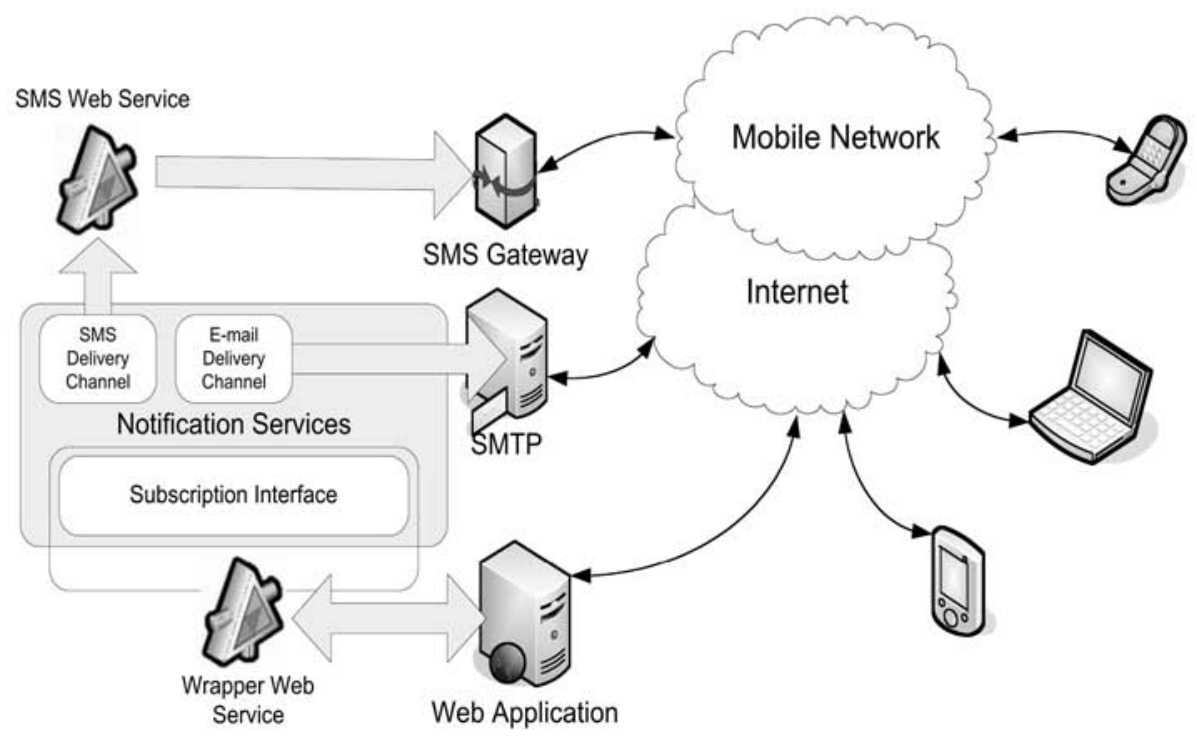

Figure 3. Overall System Architecture

\section{Discussion}

In an attempt to promote better compliance by decreasing medication errors related to dosage, frequency of administration and drug identification, various devices have been utilized. A variety of simple "pill organizers" and "unit-dose" medication systems are widely available in stores. In addition, more sophisticated devices such as human voice recordings, telephone services, and beeping watches or key chains can be used to remind the person to take medications at designated times [9]. The platform we propose goes one step further, using existing and state-of-the-art technologies, such as the .NET framework, Web Services and MS SQL Server Notification Services providing to the patient and the physician a user friendly tool that can offer notification services to both the patient and the doctor. The common web-based space shared by the patient and the physician enables the bidirectional exchange of observations or comments about the treatment, publishing additional guidelines, augmenting the treatment outcome, reporting side-effects etc. The application takes full advantage of the powerful characteristics nowadays web technologies have to offer, utilizing a system that can be accessed by any terminal (mobile or not) using a web browser without the need to purchase any additional devices. The authors of this paper believe that in the future this platform could be 
integrated with a system that uses Artificial Intelligence algorithms to detect errors regarding the medication plans issued to the patients, mining knowledge from the physician and patient reports.

Acknowledgments: This work has been co-funded

$70 \%$ by the European Union (EU) - European Regional Development Fund (ERDF)

$30 \%$ by the Hellenic Republic - Ministry of Development - General Secretariat for Research \& Technology (GSRT)

in the framework of Measure 4.2 of the Op. Pr. Competitiveness - 3rd Community Support Programme

\section{References}

1. R. B. Stewart, and L. E.Cluff, Review of medication errors and compliance in ambulant patients, Clin. Pharmacol. Ther, 13, 463 (1972).

2. I. Wandless, and J. W. Davie Can drug compliance in the elderly be improved? British Medical Journal, 1, 359-361 (1977).

3. D. Schwartz, M. Wang, L. Zeitz, M.E. Goss., Medication Errors Made by Elderly Chronically Ill Patients, American Journal of Public Health, 52, 2018-2019 (1962).

4. D. M. Parkin, British Medical Journal, 2, 686 (1976).

5. S.W. Fletcher, R.H. Fletcher, D.C. Thomas, C. Hamann, Patients' understanding of prescribed drugs, Journal of Community Health, 4 (3), 183-189 (1979)

6. P. Kanjanarat, A.G. Winterstein, T.E. Johns, R.C. Hatton, R. Gonzalez-Rothi, and Segal R. Nature of preventable adverse drug events in hospitals: a literature review. Am J Health Syst Pharm. 60:(17), 1750-1759 (2003)

7. Clickatell Bulk SMS Gateway (June 11, 2007); http://www.clickatell.com

8. Microsoft SQL Server Notification Services (June 12, 2007); http://www.microsoft.com/technet/prodtechnol/sq1/2000/evaluate/sqlnsto.mspx

9. C.A Miller., Teaching Older Adults Medication Self-Care, Geriatric Nursing 25 (5), 318-319 (2004) 